\title{
MESON CLOUDS AND NUCLEON ELECTROMAGNETIC FORM FACTORS
}

\author{
Gerald A. Miller* \\ Department of Physics, University of Washington \\ Seattle, Washington 98195-1560, USA \\ *E-mail:miller@phys.washington.edu
}

\begin{abstract}
In contrast with common non-relativistic lore, the usual Sachs form factors are not the Fourier transforms of charge or magnetization densities. Instead, the two-dimensional Fourier transform of the electromagnetic $F_{1}$ form factor is the charge charge density of partons in the transverse plane. An analysis of the available data for neutron form factors leads to the result that the neutron charge density is negative at the center, and that the square of the transverse charge radius is positive. This contrasts with many expectations. Additionally, the use of measured proton form factors leads to the result that the proton's central $u$ quark charge density is larger than that of the $d$ quark by about $80 \%$. The proton (neutron) charge density has a long range positively (negatively) charged component indicative of a pion cloud.

Keywords: Generalized Parton Distributions; Form Factors, Quark Densities
\end{abstract}

\section{Introduction}

This talk is mainly concerned with the question, "What do form factors really measure?", and secondarily with the question, "What is the relation between form factors and the orbital angular momentum of the objects that make up the neutron or proton?". The article [1], which contains more details, is the source the present presentation.

A way to focus the discussion is to ask yourself the question, "What is the charge density at the center of the nucleon?" The neutron has no net charge, but the charge density need not vanish. So we can ask, "Is the central charge density negative or positive?". This talk provides a surprising answer.

There are long-standing existing answers based on models $[2,3]$. The neutron can make a spontaneous quantum transition to a state consisting of a proton and a $\pi^{-}[2]$. The relatively light pion can spread out over a larger 
region of space than the proton. Hence one expects that the charge density is negative at the edge of the neutron and positive at the center. The same result is obtained with very different logic from the one-gluon exchange mechanism [3], which acts repulsively between two negatively charged dquarks. But enough talk about models! Here we shall be concerned with model independent information.

\section{Outline}

We shall begin with a discussion of electromagnetic form factors. This will be followed by a discussion of light cone coordinates and the relevant kinematic subgroup of the Poincaré group. Then the definitions of generalized parton distributions GPDs and a bit of math lead to the key result [4] that the two-dimensional Fourier transform of the electromagnetic form factor $F_{1}$ gives the charge density $\rho(b)$ where $b$ is the distance from the transverse center of mass, and no information about the longitudinal momentum or position is available. Then the data analysis will be discussed, and an attempt at providing an interpretation will be made. But really, finding an accurate interpretation is anyone's game.

\section{Definitions}

The electromagnetic form factors are matrix elements of the current operator, $J^{\mu}(x)$, between nucleon states of different momentum:

$$
\left\langle p^{\prime}, \lambda^{\prime}\left|J^{\mu}(0)\right| p, \lambda\right\rangle=\bar{u}\left(p^{\prime}, \lambda^{\prime}\right)\left(\gamma^{\mu} F_{1}\left(Q^{2}\right)+i \frac{\sigma^{\mu \alpha}}{2 M} q_{\alpha} F_{2}\left(Q^{2}\right)\right) u(p, \lambda),
$$

where the momentum transfer $q_{\alpha}=p_{\alpha}^{\prime}-p_{\alpha}$ is taken as space-like, so that $Q^{2} \equiv-q^{2}>0$, and $M$ is the nucleon mass. The nucleon polarization states are chosen to be those of definite light-cone helicities $\lambda, \lambda^{\prime}[5]$. The charge (Dirac) form factor is $F_{1}$, normalized such that $F_{1}(0)$ is the nucleon charge, and the magnetic (Pauli) form factor is $F_{2}$, normalized such that $F_{2}(0)$ is the anomalous magnetic moment. the Sachs form factors $[6]$

$$
G_{E}\left(Q^{2}\right) \equiv F_{1}\left(Q^{2}\right)-\frac{Q^{2}}{4 M^{2}} F_{2}\left(Q^{2}\right), G_{M}\left(Q^{2}\right) \equiv F_{1}\left(Q^{2}\right)+F_{2}\left(Q^{2}\right),
$$

were introduced to provide an expression for the electron-nucleon cross section (in the one photon exchange approximation) that depends on the quantities $G_{E}^{2}$ and $G_{M}^{2}$ but not the product $G_{E} G_{M}$. In the Breit frame, in which $\mathbf{p}=-\mathbf{p}^{\prime}, G_{E}$ is the nucleon helicity flip matrix element of $J^{0}$. Furthermore, the scattering of neutrons from the electron cloud of atoms 
measures the derivative $-d G_{E}\left(Q^{2}\right) / d Q^{2}$ at $Q^{2}=0$, widely interpreted as six times the mean-square charge radius of the neutron. However, any probability or density interpretation of $G_{E}$ is spoiled by a non-zero value of $Q^{2}$, no matter how small [1]. This is because the initial and final states have different momentum, and therefore relativistically have different wave functions. The factorization of relative and center of mass wave functions that is obtained from the non-relativistic Galilean invariance is not obtained relativistically. The internal wave function depends upon the total momentum of the nucleon. Any attempt to analytically correct for the total momentum by incorporating relativistic corrections in a $p^{2} / m_{q}^{2}$ type of expansion would be doomed, by the presence of the very light current quark mass, $m_{q}$, to be model-dependent. That is, at small values of $Q^{2}$, one finds

$$
G_{E}^{n} \sim Q^{2}\left(\int d^{3} r\left(r^{2}|\psi|^{2}+\frac{C}{m_{q}^{2}}\right),\right.
$$

where the first term represents the traditional effect depending on the square of the wave function and the unknown coefficient $C$ represents the correction due to the total momentum of the system.

\section{Light cone coordinates}

These useful coordinates involve the use of a "time"

$$
x^{+}=(c t+z) / \sqrt{2}=\left(x^{0}+x^{3}\right) / \sqrt{2} .
$$

The corresponding evolution operator is the not the Hamiltonian, $p^{0}$, but instead

$$
p^{-}=\left(P^{0}-p^{3}\right) / \sqrt{2}
$$

The orthogonal spatial coordinate is

$$
x^{-}=\left(x^{0}-x^{3}\right) / \sqrt{2} .
$$

If one quantizes at $x^{+}=0$, then $x^{-}=\sqrt{2} z$, and this why $x^{-}$is thought of as the spatial variable. The canonically conjugate momentum is given by

$$
p^{+}=\left(p^{0}+p^{3}\right) / \sqrt{2} \text {. }
$$

We note that

$$
p_{\mu} x^{\mu}=p^{-} x^{+}+p^{+} x^{-}-\mathbf{p} \cdot \mathbf{b} .
$$

The transverse coordinates perpendicular to the 0 and 3 directions are denoted as $\mathbf{b}$ and $\mathbf{p}$. 


\section{Relativistic formalism-kinematic subgroup of the Poincaré group}

The Lorentz transformation defined by a transverse velocity $\mathbf{v}$ has properties very similar to that of Galilean transformations. Under these transformations

$$
\begin{array}{r}
k^{+} \rightarrow k^{+} \\
\mathbf{k} \rightarrow \mathbf{k}-k^{+} \mathbf{v},
\end{array}
$$

and $k^{-}$transforms so that $k^{2}=k^{+} k^{-}-\mathbf{k}^{2}$ is not changed. Transverse boosts are like non-relativistic boosts according to Eq. (10).

This means that one may use $[4,7,8]$ nucleon states that are transversely localized. The state with transverse center of mass $\mathbf{R}$ set to 0 is formed by taking a linear superposition of states of transverse momentum:

$$
\left|p^{+}, \mathbf{R}=\mathbf{0}, \lambda\right\rangle \equiv \mathcal{N} \int \frac{d^{2} \mathbf{p}}{(2 \pi)^{2}}\left|p^{+}, \mathbf{p}, \lambda\right\rangle .
$$

where $\left|p^{+}, \mathbf{p}, \lambda\right\rangle$ are light-cone helicity eigenstates [5] and $\mathcal{N}$ is a normalization factor. The relevant range of integration in Eq. (11) must be restricted to $|\mathbf{p}| \ll p^{+}$to maintain the interpretation of a nucleon moving with welldefined longitudinal momentum [9]. Thus we use the infinite momentum frame, for which the nucleon may accurately be regarded as a set of a large number of partons.

\section{The main result}

Using Eq. (11) sets the transverse center of momentum of a state of total very large momentum $p^{+}$to zero, so that transverse distance $\mathbf{b}$ relative to R. can be defined. Thus we may define a useful combination of quark-field operators:

$$
\hat{O}_{q}(x, \mathbf{b}) \equiv \int \frac{d x^{-}}{4 \pi} q_{+}^{\dagger}\left(-\frac{x^{-}}{2}, \mathbf{b}\right) q_{+}\left(\frac{x^{-}}{2}, \mathbf{b}\right) e^{i x p^{+} x^{-}},
$$

where the subscript + denotes the use of only independent quark field operators. The impact parameter dependent PDF is defined [9] as the matrix element of this operator in the state of Eq. (11):

$$
q(x, \mathbf{b}) \equiv\left\langle p^{+}, \mathbf{R}=\mathbf{0}, \lambda\left|\hat{O}_{q}(x, \mathbf{b})\right| p^{+}, \mathbf{R}=\mathbf{0}, \lambda\right\rangle .
$$

The use of Eq. (11) in Eq. (13) allows one to show [1] that $q(x, \mathbf{b})$ is the two-dimensional Fourier transform of the GPD $H_{q}$ :

$$
q(x, \mathbf{b})=\int \frac{d^{2} q}{(2 \pi)^{2}} e^{i \mathbf{q} \cdot \mathbf{b}} H_{q}\left(\xi=0, x, t=-\mathbf{q}^{2}\right),
$$


with $H_{q}$ appearing because the initial and final helicities are each $\lambda$.

One finds a probability interpretation [4] by integrating $q(x, \mathbf{b})$ over all values of $x$. This sets the value of $x^{-}$to 0 , so that

$$
\int d x q(x, \mathbf{b}) \equiv\left\langle p^{+}, \mathbf{R}=\mathbf{0}, \lambda\left|q_{+}^{\dagger}(0, \mathbf{b}) q_{+}(0, \mathbf{b})\right| p^{+}, \mathbf{R}=\mathbf{0}, \lambda\right\rangle,
$$

and a density appears in the matrix elelment. If one multiplies the above relation by the quark charge $e_{q}$ (in units of $e$ ), sums over quark flavors, uses Eq. (11) with $\hat{O}_{q}(x, \mathbf{b})=e^{-i \hat{\mathbf{p}} \cdot \mathbf{b}} \hat{O}_{q}(x, \mathbf{0}) e^{i \hat{\mathbf{p}} \cdot \mathbf{b}}$ along with the sum rule relating the GPD to the form factor, the resulting infinite-momentum-frame IMF parton charge density in transverse space is

$$
\rho(b) \equiv \sum_{q} e_{q} \int d x q(x, \mathbf{b})=\int \frac{d^{2} q}{(2 \pi)^{2}} F_{1}\left(Q^{2}=\mathbf{q}^{2}\right) e^{i \mathbf{q} \cdot \mathbf{b}} .
$$

\section{Data analysis and results}

We exploit Eq. (16) by using measured form factors to determine $\rho(b)$. Recent parameterizations [11-13] of $G_{E}$ and $G_{M}$ are very useful so we express $F_{1}$ in terms of $G_{E}, G_{M}$. Then $\rho(b)$ can be expressed as an integral involving known functions:

$$
\rho(b)=\int_{0}^{\infty} \frac{d Q Q}{2 \pi} J_{0}(Q b) \frac{G_{E}\left(Q^{2}\right)+\tau G_{M}\left(Q^{2}\right)}{1+\tau},
$$

with $\tau=Q^{2} / 4 M^{2}$ and $J_{0}$ a cylindrical Bessel function. mass.

The charge density of the proton is shown in Fig. 1, and that for the neutron in Fig. 2. The proton density seems to be well determined, using the entire range of the parameterization [12], which greatly overestimates the errors, leads to little variation. The surprising feature is the negative central value of the neutron charge density. This results from the negative definite nature of $F_{1}$ [1]. The neutron density is sensitive to unknown values of $F_{1}$ at high $Q^{2}$. Cutting off the integral appearing in Eq. (17) at $Q=2 \sqrt{2} M$ leads to big changes, as shown in Fig. 2.

The neutron charge density has interesting features, as shown in Fig. 3 which displays the quantity $b \rho(b)$. It is the integral of this quantity that integrates to 0 . The neutron charge density is negative at the center, positive in the middle, and again negative at the outer edge. The medium-ranged positive charge density is sandwiched by inner and outer regions of negative charge.

One can gain information about the individual $u$ and $d$ quark densities by invoking charge symmetry (invariance under a rotation by $\pi$ about the $z$ 


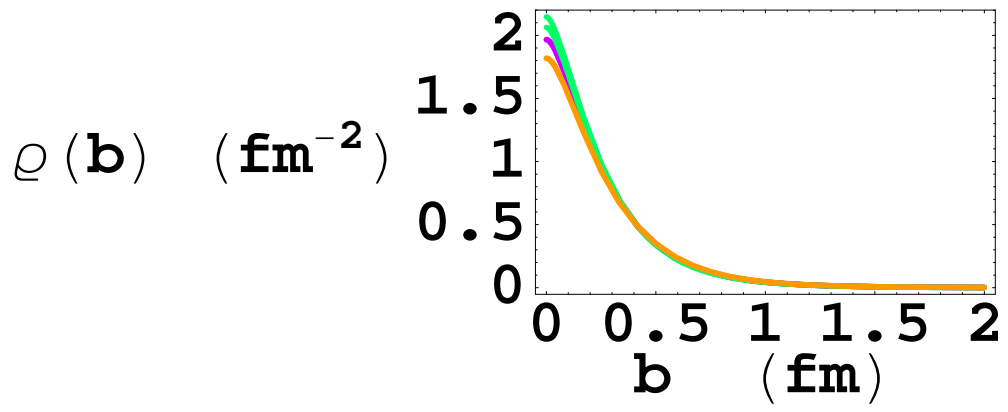

Fig. 1. The proton charge density $\rho(b)$ using the full spread of the parameters of Kelly's [12] parameterization.

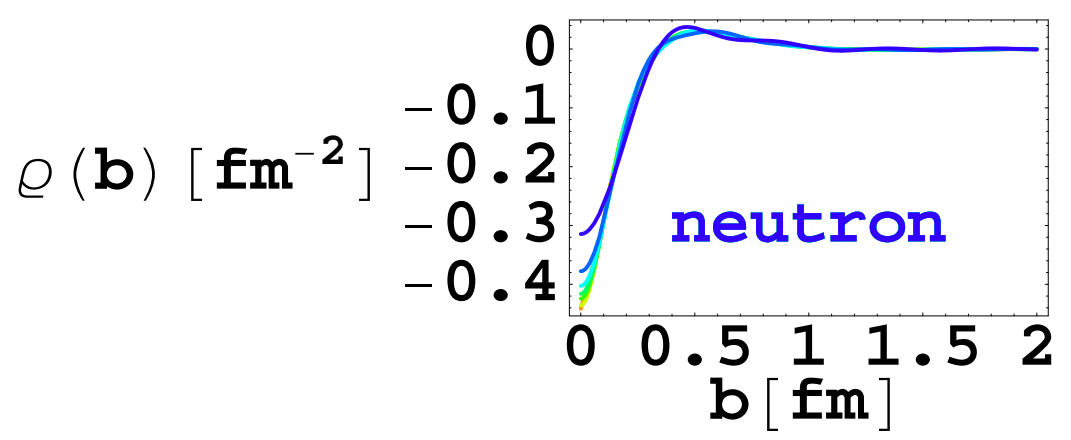

Fig. 2. The neutron charge density $\rho(b)$. The upper limit of the integral over $Q$ in the Fourier transformation Eq. (17), ranges from $Q^{2}=8 M^{2}$ to $20 M^{2}$, with the largest (least negative) value at $b=0$ obtained using $8 M^{2}$.

(charge) axis in isospin space) [14] so that the $u, d$ densities in the proton are the same as the $d, u$ densities in the neutron. We also neglect the effects of $s \bar{s}$ 
[15] or heavier pairs of quarks. In this case $\rho_{u}(b)=\rho_{p}(b)-\rho_{n}(b) / 2, \rho_{d}(b)=$ $\rho_{p}(b)-2 \rho_{n}(b)$. The results, shown in Fig. 4 , and are that the central up quark density is larger than that of the down quark by about $30 \%$.

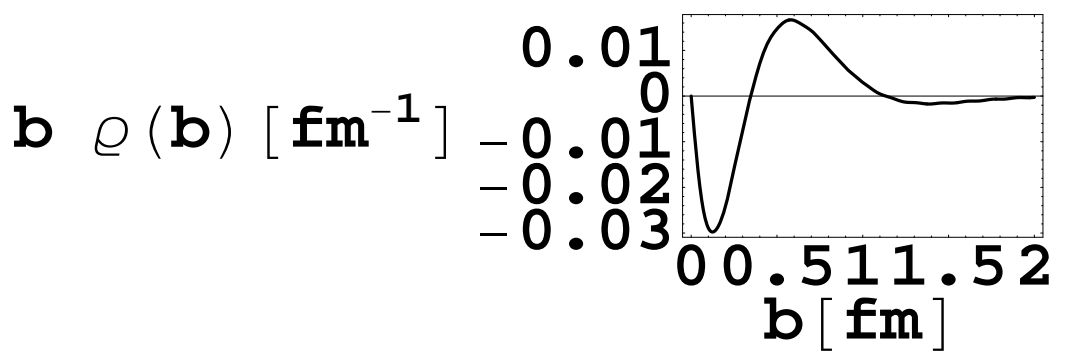

Fig. 3. The effective neutron charge density $b \rho(b)$, obtained using Kelly's [12] parameterization.

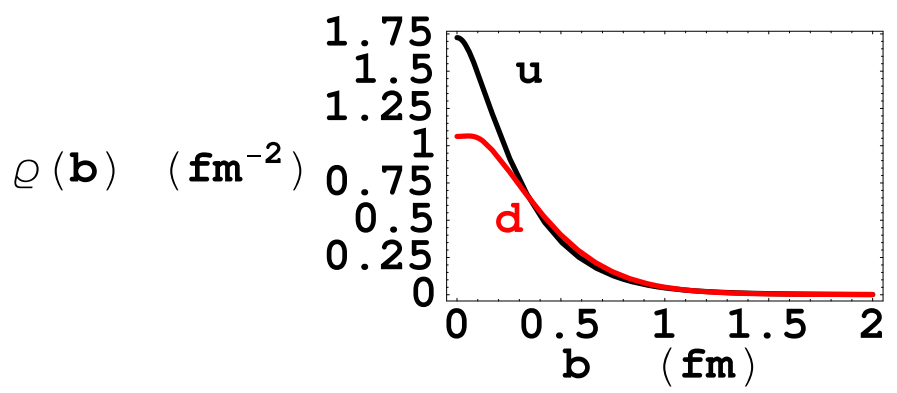

Fig. 4. The densities for $d$ (red) and $u$ (black) quarks in the proton. 


\section{Summary and Interpretation}

Model independent information about parton distributions has been obtained [1]. In particular, the central density of the neutron is negative. One possible interpretation is that quarks with high orbital angular momentum do not penetrate the interior, but the negatively charged pion, with only one unit of orbital angular momentum can both penetrate the interior and exist at long ranges. If this is the case, the negatively charged pions would be suppressed at medium range, allowing the related density to be positive.

Future measurements of neutron electromagnetic form factors could render the present results more precise, or modify them considerably. Obtaining a qualitative and intuitive understanding of our results presents a challenge to lattice QCD and to builders of phenomenological models.

\section{Acknowledgments}

This work is partially supported by the USDOE.

\section{References}

1. G. A. Miller, "Charge Density of the Neutron arXiv:0705.2409 [nucl-th], Phys. Rev. Lett. 99, 112001 (2007) .

2. A. W. Thomas, S. Théberge and G. A. Miller, Phys. Rev. D 24, 216 (1981).

3. J. L. Friar, Part. Nucl. 4, 153 (1972); R. D. Carlitz, S. D. Ellis and R. Savit, Phys. Lett. B 68, 443 (1977); N. Isgur, G. Karl and D. W. L. Sprung, Phys. Rev. D 23, 163 (1981).

4. D. E. Soper, Phys. Rev. D 15, 1141 (1977).

5. D.E. Soper, Phys. Rev. D 5, 1956 (1972).

6. R. G. Sachs, Phys. Rev. 126, 2256 (1962)

7. M. Burkardt, Int. J. Mod. Phys. A 18, 173 (2003).

8. M. Diehl, Eur. Phys. J. C 25, 223 (2002) [Erratum-ibid. C 31, 277 (2003)].

9. M. Burkardt, Phys. Rev. D 62, 071503 (R) (2000).

10. M. Diehl et al., Nucl. Phys. B 596, 33 (2001).

11. R. Bradford, A. Bodek, H. Budd and J. Arrington, Nucl. Phys. Proc. Suppl. 159, 127 (2006).

12. J. J. Kelly, Phys. Rev. C 70, 068202 (2004).

13. J. Arrington, Phys. Rev. C 69, 022201 (R) (2004).

14. G. A. Miller, A. K. Opper and E. J. Stephenson, Ann. Rev. Nucl. Part. Sci. 56, 253 (2006) G. A. Miller, Phys. Rev. C 57, 1492 (1998); G. A. Miller, A. W. Thomas and A. G. Williams, Phys. Rev. Lett. 56, 2567 (1986).

15. A. Acha et al., Phys. Rev. Lett. 98, 032301 (2007). 